\title{
BTV-vector-host interaction: the epidemiological triangle in disease transmission
}

\author{
WIESŁAW NIEDBALSKI
}

Department of Foot and Mouth Disease, National Veterinary Research Institute, Wodna 7, 98-220 Zduńska Wola, Poland

\section{Niedbalski W. \\ BTV-vector-host interaction: the epidemiological triangle in disease transmission}

\section{Summary}

Understanding the interaction between the bluetongue virus (BTV), the Culicoides vector and the ruminant host is essential to control bluetongue (BT). This triangle of interaction can be understood individually at the level of the virus, the level of vector and the host level. BTV-vector-host interactions involve physiological and ecological mechanisms, and they have evolved under a specific set of environmental conditions. Recent advances in understanding this interaction include increased knowledge of the virus replication cycle, BTV immunology and pathogenesis in the vertebrate host, as well as the virulence and pathogenicity features of newly discovered BTV serotypes. To understand the virus-host-vector interaction, new molecular biology techniques and experimental infection biology methods have been widely used. The next-generation sequencing, the establishment of a reverse genetics system for the virus, and development of novel infection models and refinement of the existing BTV experimental infection methodologies have proven very helpful. This progress in biotechnology has also made it possible to develop new-generation BTV vaccines, such as disabled infectious single cycle (DISC) vaccines and disabled infectious single animal (DISA) vaccines. However, several questions still need to be answered, such as those concerning cellular pathways involved in the induction of innate immunity and the function of NS4 in the BTV replication cycle. In addition, the identities of specific molecular determinants and the role of quasi-species diversity in determining BTV phenotype are still unclear and should be better explained.

Keywords: bluetongue virus, Culicoides vector, mammalian host, interaction

The survival and transmission of vector-borne pathogens is dependent on the interactions between the pathogen, its vertebrate host and the specific midge vector. Studies on the interactions between the vector and the vertebrate host have examined the host ranges of different vector species and determined the relative roles of different vertebrate species in supporting the haematophagous insect vector population (23). A comparatively new area of research is the study of the vector-host interface, i.e. the site of the midge attachment and feeding. It is the site at which midge-borne pathogens pass from an invertebrate to the vertebrate host system, which is a key step in the transmission dynamics and indeed the survival of the pathogen (37). Bluetongue virus (BTV) is one of the best-known examples of pathogen-vector-host interaction. Changes in the epidemiology of BTV in Europe, particularly since 1998, when the virus spread northwards into the Mediterranean Basin (36), have stimulated research on this subject.

BTV (family Reoviridae, genus Orbivirus) causes the hemorrhagic disease bluetongue (BT) in both domestic and wild ruminants. It is an infectious but non-contagious disease that causes high socio-economic and sanitary consequences. The most common signs of the disease include fever, catarrhal, stomatitis, rhinitis, enteritis and lameness (21). BT is endemic in many tropical, sub-tropical and temperate regions of the world (Africa, southern Asia, Australia, the Middle East, and the Americas), between latitudes $40^{\circ} \mathrm{N}$ and $53^{\circ} \mathrm{S}$, during times of the year that are optimal for vector activity (36). In August 2006, for the first time, BTV passed the latitude $50^{\circ} \mathrm{N}$ and disease caused by virus serotype 8 occurred in north-western Europe: the Netherlands, Belgium, Germany, France and Luxembourg. In the next two years, BTV spread to other regions of Europe, the number of outbreaks increased rapidly (about 50000 cases of disease were reported), and new BTV serotypes (BTV1 and BTV6) were detected (38). To date, twenty seven immunologically distinct serotypes of BTV have been identified worldwide (39). It is a small icosahedral virus (of about $90 \mathrm{~nm}$ in diameter) with a genome of approximately 19200 base pairs, composed of ten linear segments of double-stranded RNA (dsRNA) (28), which encode ten distinct virus proteins (35). Seven of these 
(VP1-VP7) are structural components of the icosahedral virus capsid, and three are non-structural proteins (NS1-NS3), which play different key roles during the viral replication cycle (31). BTV is almost always transmitted between its ruminant hosts by certain species of biting midges of the genus Culicoides, family Ceratopogonidae (25). These are small biting midges that occur in every inhabited continents in the world and breed in a wide variety of semi-aquatic sites, including tree holes, rotting vegetation, pond margins, damp soils and certain sorts of herbivore dung. Most Culicoides species of veterinary importance tend to breed in organically enriched, damp soil, such as is found adjacent to their hosts in and around farm holdings. There are about 1500 known Culicoides species, but only around 50 of these have been shown to be capable of developing a fully disseminated transmissible BTV infection (24). The ability of biting midges to transmit BTV is markedly influenced by ambient temperature, air humidity and total seasonal rainfall (25). It has been demonstrated that vector saliva can influence the infectivity of certain viruses (e.g. BTV), all of which appear to be more infectious in the presence of vector saliva (11). Regarding the potential effect of vector saliva on the structure and infectivity of BTV, it has been shown that treatment of BTV with proteinases, such as chemotripsin, results in the cleavage of the viral outer capsid protein VP2 and the infectious sub-viral particles. These particles demonstrate enhanced infectivity for Culicoides cells in vitro (27).

A multidisciplinary approach combining veterinary medicine, virology, entomology, immunology and biochemistry is required to fully investigate the complex BTV transmission processes and virus-vector-host interaction. Understanding the interactions between BTV, the Culicoides vector and the ruminant host is essential to control the disease. This triangle of interaction refers to (i) BTV-vector interaction, (ii) BTV-host interaction and (iii) vector-host interaction, and can be understood individually at the level of the virus, the level of the vector and the host level (Fig. 1). At the virus level, this interaction may refer to the replication of BTV in either mammalian or vector cells and includes any associated host-cell protein interactions. The ability of BTV to infect and replicate in either host or vector cells may be influenced by virus-encoded virulence factors, which may act to promote viral infection and/or inhibit the host's or the vector's antiviral responses. At the level of a Culicoides vector, the triangle of interaction may refer to the interaction of the vector with either BTV or the vertebrate host. This may include the activity of Culicoides vector-associated viral infectivity factors present in the saliva of biting midges, which may act to modify BTV proteins, thereby promoting infection of the insect, or which may suppress the host's immune response. At the host level, this triangle of interaction includes any direct or indirect pathological effects that may be induced by the virus. The pathogen-vector-host interactions involve physiological and ecological mechanisms, and they have evolved under a specific set

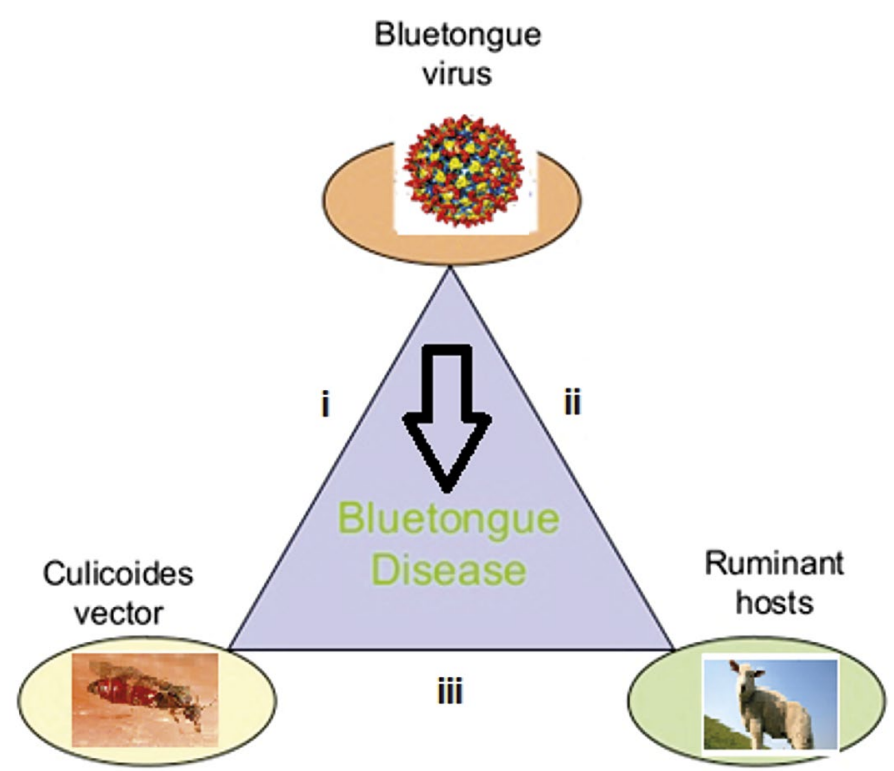

Fig. 1. The triangle of interactions between BTV, Culicoides vector and ruminant host. (i) BTV-vector interaction, (ii) BTV-host interaction, and (iii) vector-host interaction. The vertical arrow represents interactions of BTV with host-vector interface

of environmental conditions. Environmental change, therefore, will alter these interactions and, consequently, the distribution, intensity and dynamics of BT. Climate change can have direct impacts on the distribution, life cycle, and physiological status of BTV, Culicoides vectors and ruminant hosts. Climate factors can modulate BT by modifying the ecological networks to which the host-BTV-vector systems belong, and climate change can combine with other environmental stressors to induce cumulative effects on the disease (15).

Recently, several advanced laboratory techniques have been introduced to study various aspects of BT, including BTV-vector-host interaction. An increasing number of studies have developed next-generation sequencing, combined with reverse genetics (RG) and site-directed mutagenesis. Moreover, older laboratory methods, such as baculovirus expression of viral proteins in cell culture and the use of the yeast two-hybrid system, have been applied to investigate such aspects as BTV protein function and the interaction of viral proteins with host cells, and to elucidate different stages in the viral replication cycle (29). In addition, novel developments in in vitro culture systems (i.e. electrical impedance assays) have been used to investigate alternative mechanisms of disease pathogenesis, particularly those related to the role of cytokines and pro-inflammatory mediators in influencing endothelial cell dysfunction and vascular permeability (13). Traditionally, newborn mice have been used for BTV isolation and virulence studies. However, baby mice are logistically difficult to handle, so interferon alpha/beta receptor-deficient (IFNAR) mice have been developed as an alternative BTV infection model to overcome some of these problems (5).

BTV shows a complex replication cycle that can be divided into several stages, such as adsorption, viral uptake, fusion and uncoating, transcription, protein 
translation, core assembly and viral maturation. At each of these stages, the BTV proteins interact with each other and with protein components of the host cell. The viral protein domains that facilitate these interactions have been mapped in detail (29). In recent years, significant advances have been made in understanding the entry mechanisms used by BTV to enter host cells, the mechanisms by which cells are able to sense BTV infection, and the signalling pathways that lead to the induction of innate immunity and cell entry. A novel mechanism by which baby hamster kidney (BHK) cells may be infected with BTV has recently been described (16). This entry mechanism occurs independently from clathrin- or caveolae-mediated endocitosis and shares features with macropinocytosis. The mechanism by which immune cells, such as plasmocytoid dendritic cells, and non-immune cells (i.e. epithelial cells) are able to sense BTV RNA following infection, and components of the signalling pathways through which viral RNA sensing leads to the induction of interferon type 1 (IFN-1) synthesis have been recently described $(8,32)$. The replication strategy of BTV, as well as its cellular targets for replication in the mammalian host, may provide two distinct mechanisms for virus to be transmitted to the vector insect. These include the hypothesis that development of high-level viraemia is required for infection of the vector via ingestion of viraemic blood. Indeed, current control strategies are designed around the concept that a certain minimum "threshold" viraemia is required for effective virus transmission. However, replication in cells present in the skin and in mobile cells (leucocytes) that are involved in the inflammatory response to Culicoides biting, may provide another route for transmission. Further research will be required to determine whether BTV transmission to the vector can occur in the absence of a "threshold" viraemia and to clarify both BTV replication mechanisms and immune mechanisms within the ruminant host.

The essential molecular determinants that influence the virulence of BTV have been difficult to determine, because of the role of host, vector and environmental factors in influencing the severity of BT disease. The variable virulence characteristics of BTV can be related to changes in BTV properties, such as viral attachment and penetration. The rate of BTV replication, the efficiency and mechanism of viral release, together with associated membrane damage, cell death and viral spread, indicate a role of BTV proteins VP2, VP5 or NS3/ NS3a in influencing BTV virulence (18). The effects of the host species (sheep and goats) and breed, the age of individual animals, BTV serotype and host versus vector cell replication of BTV on the clinical course of BT have recently been examined (6). The results obtained indicate that the bread and age of ruminants appear to play only a small role in BT disease expression compared to, for example, differences in species susceptibility. Small differences in the virulence of viruses of different serotypes (e.g. BTV-2 and BTV-8) were found. Interestingly, it was discovered that the European strain of BTV-8 decreased in virulence during its circulation in the field. Genomic comparison of virulent BTV-8 isolated at the beginning of the BTV-8 outbreak in Europe in 2006 and BTV-8 isolated towards the end of the outbreak indicated nonsynonymous mutations in genes coding BTV proteins VP1, VP2, VP4, VP6, NS1 and NS2. The animal- and Culicoides cell-passaged virus showed increased virulence compared to virus that was passaged on BHK cells. The complete sequence of the quasi-species population in this case indicated that passage of the virus on $\mathrm{BHK}$ cells led to the reduction in the size of the population (genetic bottleneck) and reduced quasi-species diversity, whereas blood inoculums and insect cell-passaged virus demonstrated a high degree of diversity (6).

Significant advances have been made in the understanding of the pathogenesis of BT, including the explanation of the role of skin cells in BT infection, as well as the recognition that cytokines that are secreted by BTV-infected cells contribute to the pathogenesis of BT. The role of skin in supporting BTV replication has been thoroughly examined by Darpel et al (11). In these studies, an immuno-fluorescence-labelling of BTV structural and non-structural proteins VP7 and NS2 together with confocal microscopy were used to distinguish between virus presence and replication in thick skin sections from sheep. Replication was demonstrated in two cell types: vascular endothelial cells and agranular mononuclear leucocytes. The authors point out that the role of skin as a major organ for BTV replication in vivo and its role in the transmission of BTV to Culicoides has been underestimated, because of the inherent problem of extracting viral RNA from skin for molecular testing (12). The recognition of the role of skin conventional dendritic cells (cDCs) in facilitating BTV infection has also been a major development with regard to the understanding of the pathogenesis of BT. Hemati et al. (16) examined the role of ovine cDCs in BTV infection and pathogenesis and in the induction of host immune responses. For this purpose, lymph cannulation in sheep and different techniques to explore $\mathrm{cDCs}$ function were used. The results obtained indicate that BTV uses cDCs for its transportation from superficial sites of midge feeding at the skin surface to draining lymph nodes, and that lymph cDCs support production of infectious virus of different BTV serotypes regardless of the level of attenuation. The role of $\mathrm{cDCs}$ in the induction of innate immunity has been shown by the observation that cDCs respond to BTV infection by up-regulating the surface expression of T-cell co-stimulatory molecules, as well as the synthesis of cytokines involved in inflammation and immunity. BTV-infected cDCs additionally stimulated antigen-specific CD4 and CD8 cell proliferation, as well as gamma interferon production in vitro (17). In BTV pathogenesis, an important role is played by the secretion of pro-inflammatory mediators and cytokines by BTV-infected cells, in addition to direct virus-mediated damage of BTV-susceptible host cells (13). Recently, an attempt has been made to assess the relative contribution of cytokines versus direct virus-mediated injury of host 
cells in influencing BT pathogenesis in vivo in small ruminants (33). The authors used immunohistochemical techniques to examine tissues containing gross lesions from BTV-1-infected goats and evaluated co-staining of virus antigen and selected cytokines with vascular lesions. The results indicated that vascular lesions were indeed not always associated with endothelial cell destruction and viral antigen staining, but they were frequently associated with staining for selected cytokines (33).

Transmission of BTV from one animal to another is normally achieved through the bites of Culicoides midges, but under certain circumstances other routes of BTV infection can also be observed. There is some evidence for oral transmission of BTV in calves, either through contact with infected placenta or through ingestion of milk contaminated by the virus $(1,26)$. Efforts to understand the reproductive aspects of BTV-8 infection have lead to an increased understanding of BTV transplacental infection as well as the potential effects of BTV infection on the reproductive performance of ruminants. In particular, BTV-8 transplacental infection has been studied extensively in susceptible animals under both field and experimental conditions (10). Of particular note with regard to vertically transmitted infection was the finding that this infection led to the birth of relatively low numbers of viraemic offspring (2). These findings have stimulated research on potential BTV overwintering mechanisms, including studies on Culicoides survival of winter (9). Another atypical aspect of BTV-8 epidemiology in Europe was the finding that BTV was secreted at a high frequency in the semen of naturally infected rams and bulls. It was shown that BTV serotype 8 was secreted in the semen of rams and bulls of different ages at a high frequency and in the absence of blood contamination, which is unusual for a wild type strain (20). Transmission through contaminated bull or ram semen to recipient ewes or heifers has not been confirmed for BTV-8, but transmission of other BTV serotypes has been described (19).

Modified live (attenuated) virus (MLV) vaccines have been routinely used to prevent BT among sheep and cattle in many BT-affected European countries. Vaccination with MLV vaccines against BTV-2, 4, 9 and 16 has shown that some strains can cause overt disease and cause high viraemias that are sufficient for transmission of vaccine strains to Culicoides (34). Circulation of MLV strains in the field is of potential concern as MLVs may revert to virulence via genetic drift, reassortment or recombination with field strains, but supporting evidence for reversion to virulence of BTV MLVs is currently lacking (4). Inactivated BTV vaccines were developed in the 1970s and 1980s, but were not commercially available until 2005, when they were used in the field to vaccinate sheep in Corsica (France). Inactivated vaccines have several advantages over MLV vaccines: they cannot revert to virulence, reassort genes with field BTV strains or cross the placenta to cause reproductive losses. However, despite their proven efficacy and the long-term protection that these vaccines provide, their costs and the need for booster vaccination still raise problems. Since commercially available MLV and inactivated vaccines against BTV have their specific disadvantages and lack differentiation of infected from vaccinated animals (DIVA), there was a need for vaccine improvement that would solve the problems of the currently commercially available BTV vaccines. Over the last 3 decades, a variety of approaches have been investigated to develop a new type of vaccines for a broad range of BTV serotypes. Different strategies have been used to develop novel recombinant vaccines for BTV, such as baculovirus-expressed sub-unit vaccines, DNA vaccines, virus-like particles and live virus vector vaccines (30). Recently developed new-generation BTV vaccines are disabled infectious single cycle (DISC) vaccines and disabled infectious single animal (DISA) vaccines, which are able to mimic the natural tropism of the virus and to express BTV proteins at the site of infection. DISC vaccines usually contain deletion in one or more BTV genes that are essential for viral replication and are therefore avirulent, as the strains can only be grown in modified cell cultures in which the function of disabled viral proteins is provided in trans by a complementing cell line (22). In contrast, DISA vaccines are able to replicate in vivo, but, due to deletions of virulence determinants (e.g. deletions in the open reading frame of segment 10), have an avirulent phenotype and significantly reduced viraemia, making them unlikely to be transmitted in the field (14). Recently discovered BTV serotypes 25 and 26 have shown new features. BTV-25 isolated in goats in Switzerland appeared to be not pathogenic for goats, in contrast to sheep, in which infection was associated with mild clinical signs and BTV-associated pathology (7). BTV-26 isolated in sheep and goats in Kuwait in 2010 seems to indicate that goats are the natural reservoir of the virus, but no clinical signs have been observed. It has been shown that it is not possible to infect $C$. sonorensis midges with $\mathrm{BTV}-26$ by oral feeding on virusspiked blood meals, but BTV-26 transmission can occur between goats through direct contact (3).

In conclusion, it can be stated that significant progress has been made in recent years in the understanding of BTV-vector-host interaction. This includes increased knowledge of the virus replication cycle, the role of biotic factors in viral infection of the vector midges, increased knowledge of BTV immunology and pathogenesis in the vertebrate host, and increased knowledge of the virulence and pathogenicity features of newly discovered BTV serotypes and strains. Moreover, new molecular biology techniques and experimental infection biology methods have been widely applied to understand this interaction. The next-generation sequencing, the expression of individual BTV proteins in cell culture, the establishment of an RG system for the virus, the development of novel BTV infection models, and refinement of the existing BTV experimental infection methodologies have proven very helpful. These developments have also made it possible to develop a novel generation of BTV vaccines. 
However, there are still several questions to resolve, for example, with regard to cellular pathways involved in the induction of innate immunity or the function of NS4 in the BTV replication cycle. In addition, the identities of specific molecular determinants and the role of quasispecies diversity in determining BTV phenotype are still unclear, whereas the effect of virus-related vector infectivity factors on BTV transmission and BT disease severity are only now being investigated. The role of BTV evolutionary processes, including reassortment and recombination, in influencing BTV phenotype over short evolutionary time periods is also still unknown, and many key questions regarding the pathogenesis of BT remain to be addressed. The precise mechanism involved in BTV transplacental transmission and seminal shedding also requires further study. With regard to vaccinology, several new-generation vaccines against BT have been developed. However, further development and licensing of these vaccine candidates for many serotypes are needed in preparation for future BT outbreaks. To date, all novel BT vaccines developed are still under laboratory tests. They are not available commercially, and the time of their application in the field is still indefinite. Moreover, the mechanisms involved in BTV overwintering, and in particular the overwintering of BTV-8 in north-western Europe, remain unclear.

\section{References}

1. Backx A., Heutink R., Van-Rooij E., Van-Rijn P.: Transplacental and oral transmission of wild-type bluetongue virus serotype 8 in cattle after experimental infection. Vet. Microbiol. 2009, 138, 235-243.

2. Barros S. C., Ramos F., Luis T. M., Vaz A., Duarte M., Henriques M., Cruz B., Fevereiro M.: Molecular epidemiology of bluetongue virus in Portugal during 2004-2006 outbreak. Vet. Microbiol. 2007, 124, 25-34.

3. Batten C. A., Henstock M. R., Steedman H. M., Waddington S., Edwards L. Oura C. A.: Bluetongue virus serotype 26: infection kinetics, pathogenesis and possible contact transmission in goats. Vet. Microbiol. 2013, 162, 62-67.

4. Batten C. A., Maan S., Shaw A. E., Maan N. S., Mertens P. P.: A European field strains of bluetongue virus derived from two parental vaccine strains by genome segment reassortment. Virus Res. 2008, 137, 56-63.

5. Calvo-Pinilla E., Rodriguez-Calvo T., Anguita J., Sevilla N., Ortego J.: Establishment of a bluetongue virus infection model in mice that are deficient in the alpha/beta interferon receptor. PLoS One 2009, 4. doi: 10.1371/journal. pone.0005171.

6. Caporale M., Di Gialleonardo L., Janowicz A., Wilkie G., Shaw A., Savini G., van Rijn P. A., Mertens P., Di Ventura M., Palmarini M.: Virus and host factors affecting the clinical overcome of bluetongue virus infection. J. Virol. 2014, 88, 10399-10411.

7. Chaignat V., Worwa G., Scherrer N., Hilbe M., Ehrensperger F., Batten C. Cortyen M., Hofmann M., Thuer B.: Toggenburg Orbivirus, a new bluetongue virus: initial detection, first observation in field and experimental infection of goats and sheep. Vet. Microbiol. 2009, 138, 11-19.

8. Chauveau E., Doceul V., Lara E., Adam M., Breard E., Sailleau C., Viarouge C., Desprat A., Meyer G., Schwartz-Cornil I., Ruscanu S., Charley B., Zientara S., Vitour D.: Sensing and control of bluetongue virus infection in epithelial cells via RIG-I and MDA5 helicases. J. Virol. 2012, 86, 11789-11799.

9. Clausen P. H., Stephan A., Bartsch S., Jandowsky A., Hoffmann-Kohler P., Schein E., Mehlitz D., Bauer B.: Seasonal dynamics of bitting midges (Diptera: Ceratopogonidae, Culicoides spp.) on dairy farms of Central Germany during the 2007/2008 epidemic of bluetongue. Parasitol. Res. 2009, 105, 381-386.

10. Coetzee P., van Vuuren M., Venter E. H. Stokstad M.: A review of experimental infection with bluetongue virus in the mammalian host. Virus Res. 2014, 182, 21-34.

11. Darpel K. E., Langner K. F. Nimtz M., Anthony S. J., Brownlie J., Takamatsu H. H., Mellor P. S., Mertens P. P.: Saliva proteins of vector Culicoides modify structure and infectivity of bluetongue virus particles. PLoS One, 2011, 6. doi: 10.1371/journal.pone.0017545

12. Darpel K. E., Monaghan P., Simpson J., Anthony S. J., Veronesi E., Brooks H. W., Elliott H., Brownlie J., Takamatsu H. H., Mellor P. S., Mertens P. P. Involvement of the skin during bluetongue virus infection and replication in the ruminant host. Vet. Res. 2012, 43, 40. doi: 10.1186/1297-9716-43-40.
13. Drew C. P., Gardner I. A., Mayo C. E., Matsuo E., Roy P., MacLachlan N. J.: Bluetongue virus infection alters the impedance of monolayers of bovine endothelial cells as a result of cell death. Vet. Immunol. Immunopathol. 2010, 136, 108-115.

14. Feenstra F., van Gennip R. G., Maris-Veldhuis M., Verheij E., van Rijn P. A. Bluetongue virus without NS3/NS3a expression is not virulent and protects against virulent BTV challenge. J. Gen. Virol. 2014, 95, 2019-2029.

15. Gallana M., Ryser-Degiorgis M.-P., Wahli T., Segner H.: Climate change and infectious disease of wildlife: Altered interactions between pathogens, vectors and hosts. Curr. Zool. 2013, 59, 427-437.

16. Gold S., Monaghan P., Mertens P., Jackson T.: A clathrin independent micropinocytosis-like entry mechanism used by bluetongue virus-1 during infection of BHK cells. PLoS One 2010. doi: 10.1371/journal.pone.0011360.

17. Hemati B., Contreras V., Urien C., Bonneau M., Takamatsu H. H., Mertens P. P., Breard E., Sailleau C., Zientara S., Schwartz-Cornil I.: Bluetongue virus targets conventional dendritic cells in skin lymph. J. Virol. 2009, 83, 8789-8799.

18. Huismans H., van Staden V., Fick W. C., van Niekerk M., Meiring T. L. A comparison of different orbivirus proteins that could affect virulence and pathogenesis. Vet. Ital. 2004, 40, 417-425.

19. Kirkland P., Hawkes R. A.: A comparison of laboratory and "wild" strains of bluetongue virus - is there any difference and does it matter? Vet. Ital. 2004, 40, 448-455

20. Leemans J., Raes M., Vanbist T., De Clerck K., Saegerman C., Kirschvink N.: Viral RNA load in semen from bluetongue serotype 8-infected rams: relationship with sperm quality. Vet. J. 2012, 192, 304-310.

21. Maclachlan N. J.: Bluetongue: history, global epidemiology, and pathogenesis. Prev. Vet. Med. 2011, 102, 107-111.

22. Matsuo E., Celma C. C., Boyce M., Viarouge C., Sailleau C., Dubois E. Breard E., Thiery R., Zientara S., Roy P.: Generation of replication-defective virus-based vaccines that confer full protection in sheep against virulent bluetongue virus challenge. J. Virol. 2011, 85, 10213-10221.

23. Matuschka F. R., Fischer P., Heiler M., Richter D., Spielman A.: Capacity of European animals as reservoir host for the Lyme disease spirochete. J. Infect. Dis. 1992, 165, 479-483.

24. Meiswinkel R., van Rijn P., Leijs P., Goffredo M.: Potential new Culicoides vector of bluetongue virus in Northern Europe. Vet. Rec. 2007, 161, 564-565.

25. Mellor P. S., Boorman J., Baylis M.: Culicoides biting midges: their role as arbovirus vectors. Annu. Rev. Entomol. 2000, 45, 307-340.

26. Menzies F. D., McCullough S. J., McKeown I. M., Forster J. L., Jess S., Batten C., Murchie A. K., Gloster J., Fallows J. G., Pelgrim W., Mellor P. S., Oura C. A.: Evidence for transplacental and contact transmission of bluetongue virus in cattle. Vet. Rec. 2008, 163, 203-209.

27. Mertens P. P., Burroughs J. N., Walton A., Wellby M. P., Fu H., O'Hara R. S., Brookes S. M., Mellor P. S.: Enhanced infectivity of modified bluetongue virus particles for two insect cells lines and for two Culicoides vector species. Virology 1996, 217, 582-593.

28. Mertens P. P., Diprose J.: The bluetongue virus core: a nano-scale transcription machine. Virus Res. 2004, 101, 29-43.

29. Mohl B. P., Roy P.: Bluetongue virus capsid assembly and maturation. Viruses 2014, 6, 3250-3270.

30. Noad R., Roy P.: Bluetongue vaccines. Vaccine 2009, Suppl. 4, D86-89. doi: 101016/j.vaccine.2009.08.037.

31. Roy P.: Bluetongue virus proteins and particles and their role in virus entry, assembly, and release. Adv. Virus Res. 2005, 64, 69-123.

32. Ruscanu S., Pascale F., Bourge M., Hemati B., Elhmouzi-Younes J., Urien C., Bonneau M., Takamatsu H., Hope J., Mertens P., Meyer G., Stewart M., Roy P., Meurs E. F., Dabo S., Zientara S., Breard E., Sailleau C., Chauveau E., Vitour D., Charley B., Schwartz-Cornil I.: The double-stranded RNA bluetongue virus induces type 1 interferon in plasmacytoid dendritic cells via a MYD88-dependent TLR7/8-independent signaling pathway. J. Virol. 2012, 86, 5817-5828.

33. Sanchez-Cordon P. J., Pedrera M., Risalde M. A., Molina V., Rodriguez-Sanchez B., Nunez A., Sanchez-Vizcaino J., Gomez-Villamandos J. C.: Potentia role of proinflammatory cytokines in the pathogenic mechanisms of vascular lesions in goats naturally infected with bluetongue virus serotype 1 . Transbound Emerg. Dis. 2012, 60, 252-262

34. Veronesi E., Darpel K. E., Hamblin C., Carpenter S., Takamatsu H. H., Anthony S. J., Elliott H., Mertens P. P. Mellor P. S. Viraemia and clinical disease in Dorset Poll sheep following vaccination with live attenuated bluetongue virus vaccines serotypes 16 and 4. Vaccine 2010, 28, 1397-1403.

35. Verwoerd D. W.: History of orbivirus research in South Africa. J. S. Afr. Vet. Assoc. 2012, 83:532. doi: 10.4102/jsava.v83i1.532

36. Walton T. E.: The history of bluetongue and a current global overview. Vet. Ital 2004, 40, 31-38

37. Wikel $S$. K.: Tick modulation of host immunity, an important factor in pathogen transmission. Int. J. Parasitol. 1999, 29, 851-859.

38. Wilson A. J., Mellor P. S.: Bluetongue in Europe: past, present and future. Philos Trans. R. Soc. Lond. B. Biol. Sci. 2009, 364, 2669-2681.

39. Zientara S., Sailleau C., Viarouge C., Höper D., Beer M., Jenckel M., Hoffmann B. Romey A., Bakkali-Kassimi L., Fablet A., Vitour D., Breard E.: Novel bluetongue virus in goats, Corsica, France, 2014. Emerg. Infect. Dis. 2014, 20, 2123-2125.

Corresponding author: dr hab. Wiesław Niedbalski, assoc. prof., Wodna 7 98-220 Zduńska Wola, Poland; e-mail: wieslaw.niedbalski@piwzp.pl 